\title{
RANS study of very high Reynolds-number plane turbulent Couette flow
}

\author{
A. Sherikar and P. J. Disimile \\ Department of Aerospace Engineering and Engineering Mechanics, CEAS, University of Cincinnati, Ohio, USA. \\ Phone: +1 513-556-3548; Fax: +1 513-556-5038.
}

\begin{abstract}
The objective of this study is to expound on the deliverables of a steady-state RANS (Reynolds Averaged Navier Stokes) simulation in one of the simplest flows, Couette flow, at a very high Reynolds number. To that end, a process to perform better grid sensitivity testing is introduced. Three two-equation turbulence models (Std $k-\varepsilon$, Std $k-\omega$, and $k-\omega S S T$ ) are compared against each other as well as pitted against formal literature on the subject and core flow velocities, slopes, wall-bounded velocities, shear stresses and kinetic energies are analyzed. Std $k-\varepsilon$ applied with enhanced wall functions is consistently found to be in better agreement with previous studies. Finally, plane turbulent Couette flow at $\operatorname{Re} 51,099$, the range at which it has not been studied experimentally, numerically or analytically in former studies, is simulated. The results are found to be consistent with the trends asserted by literature and preliminary computations of this study.
\end{abstract}

ARTICLE HISTORY

Revised: $28^{\text {th }}$ Jan 2020

Accepted: 27th Feb 2020

\section{KEYWORDS}

Turbulent flows;

Couette flow;

RANS;

turbulence models;

high Reynolds number.

\section{INTRODUCTION}

A Couette flow is realized when two surfaces in relative motion with respect to each other and separated by a finite distance drive a viscous fluid between them by a pure shearing action. The simplest form is a plane Couette flow, where the bounding surfaces are flat and parallel to each other. With the exception of lubricating and thin film flows, Couette flow is generally turbulent in nature and requires a separate focus of study. Due to the moving wall, though it is considered to be a simple flow, Couette flow is difficult to setup experimentally. In fact, in his seminal publication in 1959, Robertson [1] stated that until then, "(the flow) had never been achieved. In fact, there was even some doubt whether turbulent plane Couette flow could exist." Until 1959, the only remarkable experimental study on plane Couette flow was Reichardt's study in 1956 [2]. These studies [1,2] focused on gross flow characteristics like velocity profiles across the channel, wall shear stress and the coefficient of friction. Their flow Reynolds numbers ranged from 4,800 to 17,000. Leutheusser, in his studies of transition in plane Couette flow in 1971 [3] and 1979 [4], pushed the envelope further in the laminar regime publishing results for flows with Reynolds number as low as 230. In 1982, for the first time, El Telbany and Reynolds [5] recorded the profiles of $v^{\prime}$ and $w^{\prime}$ apart from $u^{\prime}$ as well as time-mean velocity, turbulence kinetic energy and production of turbulence energy across the channel [5]. Reynolds numbers for their study ranged from 9,500 to 19,000. This publication provided the turbulence research community with experimental results to verify analytical and theoretical studies conducted over 90 years. In the next few years, up until the mid-1990s, many studies focused on transition in Couette flow. Towards this effort, several authors such as Tillmark and Alfredsson [6] conducted flow visualization experiments in plane Couette flow. They determined that the turbulence could be sustained for a Reynolds number as low as 360. In the experimental investigations listed till the end of the millennium, the highest Reynolds number Couette flow was that documented by El Telbany and Reynolds [5] at 19,000.

A cursory review of research from 1950s to the late 1990s suggests that turbulent boundary layers and generic channel flows were subject to many CFD investigations. Until the late 1990s, dedicated RANS study of Couette flow was limited and could be found in the works of the following authors: Henry and Reynolds [7] applied $k-\varepsilon$ and $k-\omega$ models by reducing the model equations to gradient-diffusion models, and then solved them analytically. The reduced model equations were applicable only to high Reynolds numbers, so they needed wall functions to approximate variables at the wall where the Reynolds numbers are very low. They found that the turbulence kinetic energy was uniform and concluded that its production balanced the dissipation over the entire region. In 1994, Andersson and Pettersson [8] published results of a second moment closure study of a plane turbulent Couette flow. They recorded some earlier attempts of employing closure models to simulate the flow namely Nisizima and Yoshizawa [9] who in 1987 adopted an anisotropic $k-\varepsilon$ closure for the whole computational domain but failed to reproduce the near wall peaks shown by experimental studies [8]. Additionally, between 1987 to 1993, many authors like Monnier and Stanislas [10], Zhang et al. [11] and Sund [12] tried to obtain results by employing second moment closure methods and other modifications but were unsuccessful in matching with the experiments. In all the studies mentioned, the highest Reynolds number pure Couette flow studied in any form was limited to 19,000 and was attributed to El Telbany and Reynolds [5] back in 1982.

In the last 20 years, with advancements in DNS techniques, research on plane turbulent Couette flow has proliferated and brought novel flow attributes into focus. Scale-by-scale transport interactions and dynamic phenomena like laminar- 
turbulent transition, coherent structures and banded patterns [13] are extensively being investigated. While the existence of secondary large scale coherent structures was first reported in a DNS study by Lee and Kim [14] in 1991, in the recent years, they have been the subject of investigations in various forms. Pershin et al. [15] reported on the spatially localized structures in plane Couette flow that play an important role in transition to turbulence. Eckhardt and Zammert [16] used the dynamical systems theory to study the contribution of secondary bifurcations in the cascading of the exact-coherentstates to smaller scales while Olvera and Kerswell [17] studied the existence of exact-coherent-structures in stably stratified plane Couette flow. Kawata et al. $[18,19]$ studied the inter-scale Reynolds shear stress transport in turbulent plane Couette flow. Contrary to initial estimate that these coherent structures would be averaged out because of time averaging, the fact that these structures are weakly dependent on time suggests that a RANS study may be capable of simulating them. This possibility was explored successfully by Spalart et al. [20] by employing the Spalart-Allmaras model (one-equation eddy-viscosity model) in conjunction with Quadratic Constitutive Relation (QCR). Guo [21] proposed a general velocity distribution law taking into account the existing knowledge of theory, experiments and DNS simulations and introducing a rational eddy-viscosity model. Similar efforts at data driven analysis as well as the use of machine learning in turbulence modeling is growing and finding its place among the tools available for fluids research $[22,23]$. Towards the effort of increasing the envelope of high Reynolds number simulated, Hoyas and Jimenez [24] published their findings of DNS simulation of $R e_{\tau}$ of 2003 while they reported only on the scaling of the velocity fluctuations. Mortikov et al. [25] conducted DNS simulations of Reynolds number in the range of 2,600 - 40,000. Pirozzoli et al. [26], in 2014, investigated Couette flow using a DNS simulation and reported a Reynolds number of 21,333 corresponding to $R e_{\tau}$ of 986 .

Any scientific investigation, be it experimental, analytical, computational or data driven, has its advantages and limitations. The hurdle with a DNS study is the significant amount of computational resources and time it requires. The problem is further complicated as the flow Reynolds number increases because the simulations require proportionally finer grids. It pushes the computation time and number of operations to grow at the rate of $R e^{\frac{9}{4}}$ in a $3 \mathrm{D}$ domain. For an incompressible study, the relative velocities of the wall must be under Mach 0.3 to avoid effects of compressibility. It greatly restricts the highest Reynolds number that can be achieved. To circumvent this problem, the flow domain can be made wider at the cost of exponentially increasing computational resources. The flow domain can be widened only to an extent where the flow bounding surfaces are still coupled. An experimental study would require a physical setup with many flow parameters carefully monitored for reliable results. Despite these efforts, uncertainty induced by each parameter needs to be carefully accounted for. Analytical studies provide great insight into the flow physics because the solutions are mathematical in nature, but they are a result of the assumptions and approximations made in the process of finding them [27]. Hence, in the study of turbulent flows which contains many such approximations, the solutions are limiting in a way that they may apply only to a niche set of flows where the assumptions are valid. Additionally, real world flow regimes are affected by many external factors. To account for them in an analytical study is infeasible. Data driven models are better equipped to handle a multitude of factors affecting the flow regime, but they are only as good as the data and the learning algorithms they are trained with.

Given that the demand for new information is absolute in any pursuit, academic or commercial, the application of every investigative tool becomes imperative. To that end, with new potential for Couette flow applications made possible by emerging high-speed technologies like the Hyperloop and the Rail-Gun project among others as general motivation, plane turbulent Couette flow is revisited for RANS analysis in a range of Reynolds number much higher than past studies.

The objective of the current study is to arrive at a reliable computational parameter space that can be used in a RANS study and to validate against available experimental and DNS data, and thereby provide confidence that these simulations can reliably predict gross flow characteristics at higher Reynolds number, when compared against literature.

\section{TURBULENCE MODELING}

Navier Stokes equations can describe any given fluid flow field completely. To solve them numerically, their nonlinear nature and flow domain discretization pose computational challenges, especially when there is a wide range of scales of motion to be resolved. Since turbulent flow is comprised of a range of scales varying in orders of magnitude, a numerical description of turbulence becomes very difficult to realize. Hence, some length and velocity scales associated with turbulent flow are averaged for a gross study of turbulent behavior. Statistical calculation methods are used to average out and approximate turbulent behavior resulting in RANS.

The way turbulence is modeled plays a crucial role in determining the accuracy of the simulation. Sometimes turbulence models are constructed with emphasis on theoretical concepts, which in themselves involve gross assumptions about the turbulent nature of the flow. Commercial CFD software provide us with many parameters that need to be specifically tuned to the flow to achieve the most reliable solution. It is often unclear whether improvements made in a turbulence model for one class of flows would be equally reliable for other class of flows. Hence, this study is aimed at systematic establishment of the turbulence model and related parameters as we move towards better solution accuracy for turbulent Couette flow. 


\section{Turbulence Model and Near Wall Treatment}

$k-\varepsilon$, and $k-\omega$ models along with their variants form a part of a category of two-equation models that first described turbulence fully - in that they solved independent transport equations for both the turbulence length scale, or some equivalent parameter, either $\varepsilon$ (turbulence dissipation) or $\omega$ (rate or frequency of turbulence dissipation) and the turbulent kinetic energy $k$ [28]. These models assume a balance between turbulence production and dissipation which leads to an assumption of a local equilibrium and are known to work well with a large variety of flows where this assumption is not grossly violated. They also assume that the turbulent fluctuations are isotropic, which is true for smaller eddies at high Reynolds numbers. It makes them suitable for simulating free shear flows seen in jets and wakes [28]. An advantage of a shear stress transport model (SST) of the $k-\omega$ family is that it accounts for the transport of the turbulent shear stress. While this quantity is individually calculated in the Reynolds-stress models, the SST model computes it from turbulent viscosity and the velocity gradient [29].

Flows very close to the wall are subject to several different effects in that the Reynolds number very close to the wall tends to zero and the velocity gradients are very high giving rise to a very high shear [28]. Here, turbulence tends to its two-component limit and as a consequence of the no-slip condition, flow up to the integral scale is affected [28]. Neither $k-\omega$ nor the $k-\varepsilon$ models reproduce theoretical near wall trends, but the $k-\omega$ SST model has known to come close enough in predicting law of the wall when modeling the viscous sublayer without significant error [28]. Std $k-\varepsilon$ cannot model the viscous sublayer without corrections. To ease the expense of numerical calculations in a distinct and complex flow regime such as near the wall, wall functions were developed as an additional calculation to $k-\varepsilon$ models [30]. Wall functions generally comprise of a set of formulae that model scalars to follow the law of the wall and nearwall turbulent quantities [31]. Literature from ANSYS Fluent classifies the available wall functions into standard, nonequilibrium and enhanced wall functions. Standard wall functions generally model the logarithmic part or turbulent regime of the boundary layer $\left(y^{+}>30\right)$. The non-equilibrium type generally divides the whole turbulent boundary layer starting from the viscous sublayer, the buffer layer up to the turbulent regime into two layers namely the viscosity affected region and the turbulent region. Enhanced wall functions formulate the law of the wall as a single wall layer for the entire boundary layer region. ANSYS Fluent achieves this by blending the linear (viscous) and the logarithmic (turbulent) law of the wall using one function [31]

In this study, the focus is on three models - the Std $k-\varepsilon$ with Enhanced Wall functions, Std. $k-\omega$ and $k-\omega S S T$ models.

\section{COMPUTATIONAL STRATEGY}

Pirozzoli et al. [26] investigated turbulent Couette flow at high Reynolds numbers through a series of large-scale DNS for Reynolds numbers, $R e=h u_{w} / 2 v$, of 3000,10,133 and 21,333, where $h$ is channel half height, $u_{w}$ is the velocity of the top plate. In this study, RANS computations were run for the same Reynolds numbers and results were compared for validation. Before validation, a series of sets of simulations were completed in a hierarchy starting with a grid sensitivity study. Table 1 documents the details of these sets of simulation. The objective of each set drives the design of the simulations in that set. Results from all previous simulation sets are used to determine parameters that are used in the simulation set aimed at validation. Sets A and B are aimed at grid sensitivity study, the details of which are discussed in the sections that follow. Set $\mathrm{C}$ is aimed at determining whether the flows in two different flow configurations are equivalent. Set D is aimed at deciding, by verification against available literature, the turbulence model that needs to be used for simulating plane turbulent Couette flow. Set $\mathrm{E}$ is aimed at validating the turbulence model decided in set D against three Reynolds numbers chosen from [26]. Set F consists of the simulation where the highest Reynolds number, $R e=51,099$, is simulated and studied.

Table 1. Details of simulation sets used for the current study.

\begin{tabular}{|c|c|c|c|c|c|c|c|}
\hline $\begin{array}{c}\text { Simulation } \\
\text { set }\end{array}$ & $y^{+}$ & {$\left[\Delta x_{c}, \Delta y_{c}\right] \mu m$} & $\begin{array}{c}\text { Turbulence } \\
\text { model }\end{array}$ & Plate movement & $u_{w}(\mathrm{~m} / \mathrm{s})$ & $\begin{array}{l}\text { Reynolds } \\
\text { number } \\
\text { simulated }\end{array}$ & Aim of Simulation \\
\hline $\begin{array}{l}\text { A1, A2, A3, } \\
\text { A4, A5, A6, } \\
\text { A7, A8 }\end{array}$ & $\begin{array}{c}1,0.5 \\
0.25, \ldots, \\
0.0078125\end{array}$ & {$[500,500]$} & $S t d . k-\varepsilon$ & Top plate & 49.84 & 21,333 & $\begin{array}{c}\text { Grid sensitivity } \\
\text { study }-y^{+} \\
\text {refinement }\end{array}$ \\
\hline $\begin{array}{c}\text { B-1, } \\
\text { B0(=A3), } \\
\text { B1 }\end{array}$ & 0.25 & $\begin{array}{c}\text { B-1:[1000, 1000] } \\
\text { B0: }[500,500] \\
\text { B1: }[250,250]\end{array}$ & Std. $k-\varepsilon$ & Top plate & 49.84 & 21,333 & $\begin{array}{l}\text { Grid sensitivity } \\
\text { study - Overall } \\
\text { grid refinement }\end{array}$ \\
\hline $\mathrm{C} 1, \mathrm{C} 2$ & 0.25 & {$[500,500]$} & Std. $k-\varepsilon$ & $\begin{array}{c}\text { C1: Top plate } \\
\text { C2: Both plates }\end{array}$ & $\begin{array}{l}\mathrm{C} 1: 49.84 \\
\mathrm{C} 2: 24.92\end{array}$ & 21,333 & $\begin{array}{c}\text { Test if } \mathrm{C} 1 \text { and } \mathrm{C} 2 \\
\text { are equivalent }\end{array}$ \\
\hline
\end{tabular}




\begin{tabular}{|c|c|c|c|c|c|c|c|}
\hline $\mathrm{D} 1, \mathrm{D} 2, \mathrm{D} 3$ & 0.25 & {$[500,500]$} & 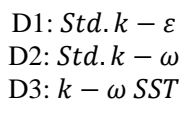 & Top plate & 49.84 & 21,333 & $\begin{array}{l}\text { Turbulence model } \\
\text { study }\end{array}$ \\
\hline E1, E2, E3 & 0.25 & {$[500,500]$} & Std. $k-\varepsilon$ & Top plate & $\begin{array}{l}\text { E1: } 7.00 \\
\text { E2: } 23.67 \\
\text { E3: } 49.84\end{array}$ & $\begin{array}{l}\text { E1: } 3,000 \\
\text { E2: } 10,133 \\
\text { E3: } 21,333\end{array}$ & Validation \\
\hline F1 & 0.25 & {$[500,500]$} & Std. $k-\varepsilon$ & Top plate & 119.4 & 51,099 & $\begin{array}{l}\text { Higher Reynolds } \\
\text { number case }\end{array}$ \\
\hline
\end{tabular}

\section{Geometry}

Couette flow between two infinite plates is shown in the Figure 1. Since the flow is identical in the z direction, a 2D rectangular domain in the $x-y$ plane at an arbitrary cross section is chosen for simulations. The domain's length and height are chosen to be $L_{x}=0.05 \mathrm{~m}$ and $h=0.0125 \mathrm{~m}$ respectively. Infinite plate condition is modeled by applying a periodic boundary condition on the inlet and outlet. Figure 1(a) shows the flow geometry in 3D, where the plates in pink and blue respectively extend infinitely in the z-x plane. Figure 1(b) shows the rectangular computational domain considered for the current study. It is constructed by slicing the $3 \mathrm{D}$ geometry along the $\mathrm{x}-\mathrm{y}$ plane at an arbitrary location on $\mathrm{z}$ axis.

\section{Boundary Conditions}

Since the domain is a simple rectangle and the flow being simulated is a steady turbulent flow, we specify timeindependent values on its four walls (Dirichlet boundary conditions). Relative motion between the top and the bottom plates can be simulated by one of the two configurations: 1$)$ only the top plate moving with a plate velocity $\left(u_{w}=2 u_{c}\right)$ and bottom plate stationary, 2 ) top and bottom plates moving in opposite directions with centerline velocity $\left(u_{c}\right) . u_{c}$ (tabulated in Table 1) is determined by the Reynolds number, $R e$, for the flow to be simulated. The inlet and outlet faces are imparted with periodic boundary condition with zero pressure gradient to simulate infinite plate condition. A periodic boundary condition replicates the values computed at the inlet on the outlet suggesting that the flow occurring at both these surfaces is equivalent thereby simulating an infinite condition. Figure 2 illustrates the boundary conditions on the two configurations.

[a]

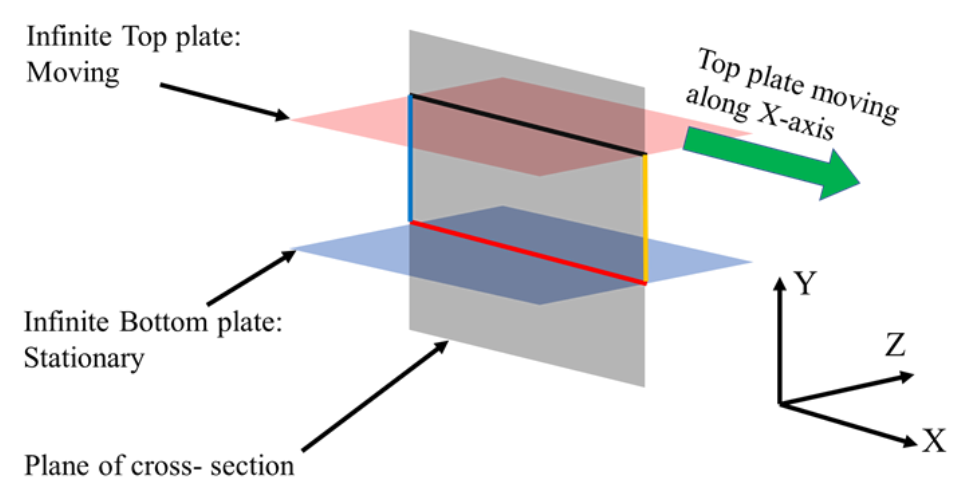

[b]

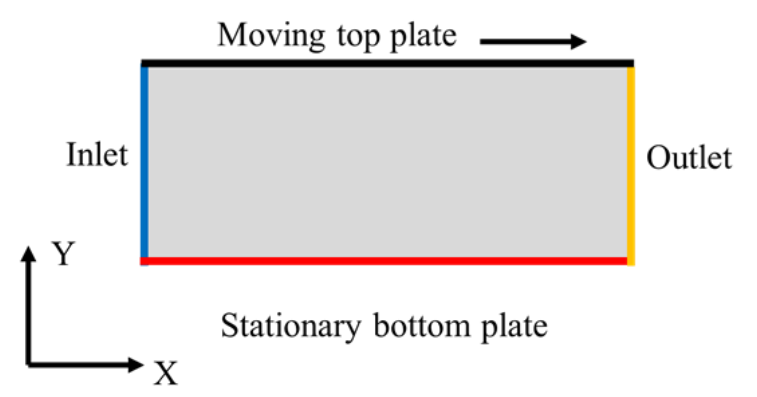

Figure 1. Flow geometry. 
[a]

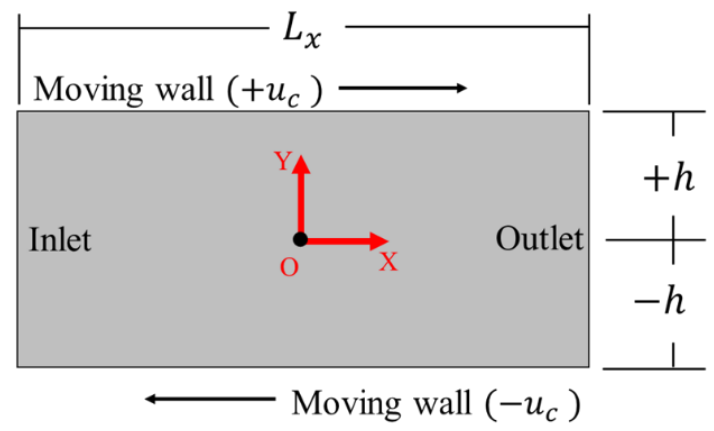

[b]

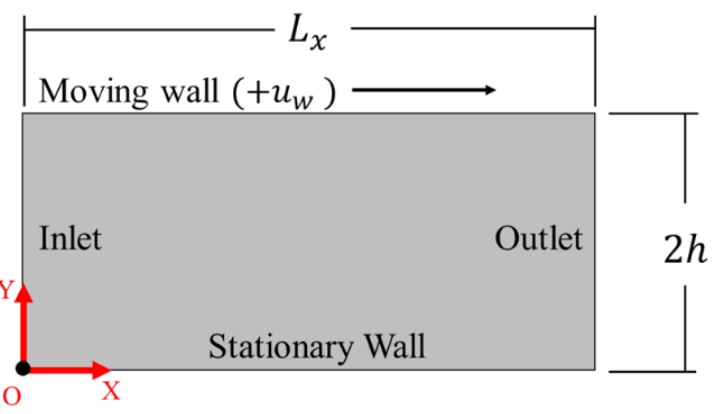

Figure 2. Flow configurations for $\mathrm{C} 1$ and $\mathrm{C} 2$ : (a) is used for simulation $\mathrm{C} 2$ and (b) is used for simulation $\mathrm{C} 1$.

While we may intuitively understand relative motion and its effects in solid mechanics, it may not be the case when studying fluid dynamics. For this study, initially, Couette flow is modeled differently by two simulations C1 and C2 with a different set of absolute velocities for the walls. It is hypothesized that a difference in results may arise because the shearing of fluid starts at both walls in case $\mathrm{C} 2$ but only on the top wall in the case $\mathrm{C} 1$. Another way to conceptualize the difference is to suppose that the top and bottom walls have different roughness or waviness. The difference in results arising from shearing action initiated at different walls may now be immediately apparent. The focus of studying simulations $\mathrm{C} 1$ and $\mathrm{C} 2$ is to not read into the flow parameters that define the flow but regard them as mere variables that are needed for a computational solution of the flow domain. Subsequently, results from the two simulations are analysed to check if they converge despite two different boundary conditions being applied.

Normalized velocity profiles and higher order variables like turbulence kinetic energy and turbulence dissipation rate across the domain height were plotted for simulations $\mathrm{C} 1$ and $\mathrm{C} 2$. The average shear stress on the walls for both $\mathrm{C} 1$ and $\mathrm{C} 2$ is computed to be $1.475 \mathrm{~Pa}$. Figure 3 and the recorded wall shear stress amply suggest that simulations $\mathrm{C} 1$ and $\mathrm{C} 2$ are equivalent. The boundary conditions in $\mathrm{C} 1$ and $\mathrm{C} 2$ were defined in such a way that the walls' relative velocities were same, and hence the associated Reynolds number, remained the same. It also reinforces the importance and the implications of defining a Reynolds number - a unique non-dimensional number, which could be achieved by any combination of flow parameters. This fact could be leveraged to facilitate study in cases where certain flow parameters or geometries are restricted in any way. To clarify, this study assumes that the domain-confining walls are smooth, and, that any instantaneous time-dependent flow attributes introduced by turbulence will be averaged out by Reynolds averaging. Hence the difference between the two simulations may have remained untraceable. Higher fidelity simulations or experimental investigations may be needed to track such differences. 
[a]

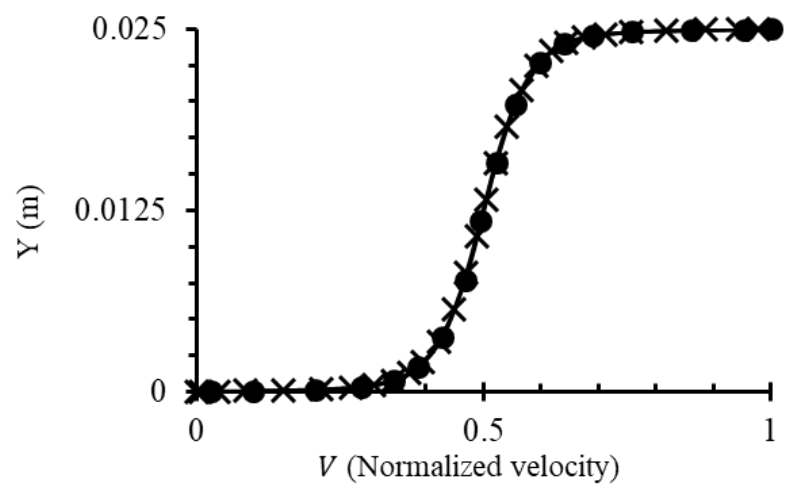

[b]

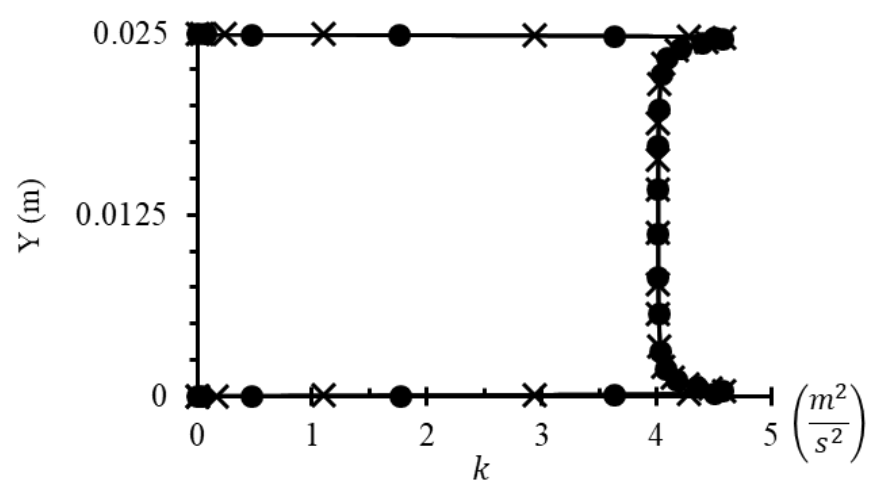

[c]

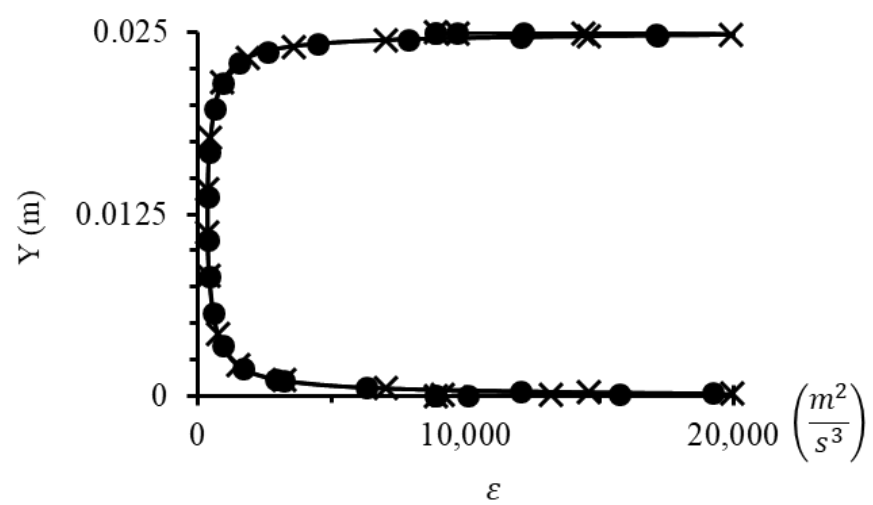

Figure 3. Comparison of simulations C1 (solid circles) and C2 (cross-marks).

To summarize, in purview of simple geometries like that of a plane Couette flow of a Newtonian fluid where roughness of the walls is not considered, RANS simulations $\mathrm{C} 1$ and $\mathrm{C} 2$ are equivalent for all analysis purposes. Since the time taken to run a simulation is majorly dependent on the grid, discretizing scheme and the turbulence model used, there is no effect on runtime and computational resources due to change in boundary conditions. However, this result gives us the freedom to conceptualize a flow in a manner that best fits our computational interests.

\section{GRID}

For this study, a characteristic grid is defined as a structured Cartesian 2D grid made up of identical mesh cells applied throughout the rectangular domain in the absence of inflation layers (defined further). Grid layer inflation is applied to the characteristic grid near the walls to distort it in a way to increase the height of subsequent layers in geometric progression. This is done to capture high gradients of velocity, pressure and other turbulence quantities in viscous and buffer layers near the wall. Starting with the first layer adjacent to the wall, inflation layers grow to characteristic cell height, $\Delta y_{c}$, based on the number of inflation layers $(n)$ and their growth ratio $\left(r_{\text {inf }}\right) . \Delta y_{c}$ is determined by the overall grid refinement study. The inflation layers are deployed on the top and the bottom walls. A schematic illustration of the grid used in this study is shown in Figure 4. Figure 4(a) shows the characteristic cell with its dimensions while Figure 4(c) shows the inflation layers applied to the mesh at the walls of the domain. 


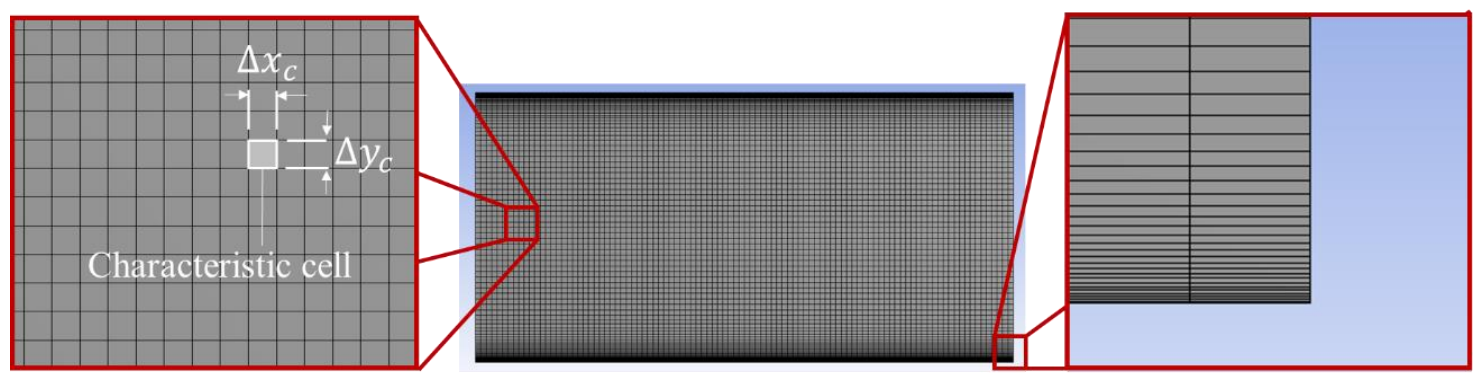

[a]

[b]

[c]

Figure 4. Schematic illustration of mesh used for current study.

\section{GRID SENSITIVITY STUDY}

Generating a suitable mesh for a flow domain is critical in obtaining a reliable solution. It is generally obtained by tinkering various parameters such as cell structure (structured/ unstructured), mesh resolution, inflation layers, aspect ratio among other factors. For a careful and meticulous study of the effect of grid on the solution, a novel approach is conceived. In this approach we separately concentrate on the effect of two factors namely characteristic grid size $\left(\Delta y_{c}\right)$ and inflation layers. The characteristic grid size decides if there is enough resolution of uniformly distributed grid points in the whole domain to capture the required variables. The biggest cell size may be limited by the minimum resolution required and whether the aspect ratio of the cells is within the acceptable limits when inflation layers are growing to characteristic grid size. The smallest cell size may be limited by computational resources and time taken for the simulation. Inflation layers are responsible for transitioning from a fine grid near the walls to size of the characteristic grid. Turbulence model and wall functions dictate the size of $y^{+}$. It is critical to control the growth of the layer height from $\Delta y_{1}$ to $\Delta y_{c}$ and hence the inflation growth ratio, $r_{\text {inf }}$ generally is chosen between 1 and 1.2.

With these constraints at hand, the approach suggested in the following two sections is used to gauge the effect of these two parameters separately. The grid sensitivity study is carried out by controlling the parameters: $y^{+}$(in Section named 'Refinement of $y^{+}$') and $\Delta y_{c}$ (in Section named 'Overall Grid Refinement') separately and independently while keeping the other as a constant to avoid a compounded effect of changing two parameters. The aspect ratio $A R$ is kept constant at 1 , which requires changing $\Delta x_{c}$ accordingly.

\section{Refinement of $y^{+}$}

The choice of turbulence model and accompanying wall functions determine the minimum $y^{+}$and in effect, the value of $\Delta y_{1}$ required by the simulation. While the minimum value is necessary, the Reynolds number of a simulated flow may dictate how small a $y^{+}$is needed to accurately model the viscous sublayer. Hence a set of simulations (A1, A2, .., A8) were run with a $y^{+}$starting from 1 and down until 0.0078125 in steps of geometric ratio of 0.5 while $\Delta y_{c}$ is kept constant at 500 microns. The number of inflation layers, $n$, is different for each simulation as the inflation layers must grow from a different value of $\Delta y_{1}$ in each simulation to a constant $\Delta y_{c}$ with $r_{\text {inf }}=1.1$. The specifics are tabulated in Table 2. Since $A R$ is kept constant at $1, \Delta x_{c}$ remains 500 microns for this study.

Table 2. Details of $\mathbf{y}^{+}$refinement study.

\begin{tabular}{ccccc}
\hline Simulation & $y^{+}$ & $\Delta y_{1}$ (microns) & $r_{\text {inf }}$ & $n$ \\
\hline A1 & 1 & 10 & 1.1 & 41 \\
A2 & 0.5 & 5 & 1.1 & 48 \\
A3 & 0.25 & 2.5 & 1.1 & 55 \\
A4 & 0.125 & 1.25 & 1.1 & 62 \\
A5 & 0.0625 & 0.625 & 1.1 & 70 \\
A6 & 0.03125 & 0.03125 & 1.1 & 77 \\
A7 & 0.015625 & 0.015625 & 1.1 & 84 \\
A8 & 0.0078125 & 0.0078125 & 1.1 & 91 \\
\hline
\end{tabular}


The values of wall shear stress on the top and the bottom plates are plotted against decreasing $y^{+}$values. The velocity profiles of all simulations in the set shown in Figure 5(a) match with each other. To resolve the decision of $y^{+}$, we consider a higher order variable - the wall shear stress. Since the flow is symmetric, we expect the values of wall shear stress to be equal on both the plates. In Figure 5(b), we see this is true for $y^{+}$of 0.25 . On refining $y^{+}$, the value tends to oscillate and return to the value projected by $y^{+}$of 0.25 . Hence, simulation A3 with a $y^{+}$of 0.25 is chosen for further simulations in favor of computational resources available.
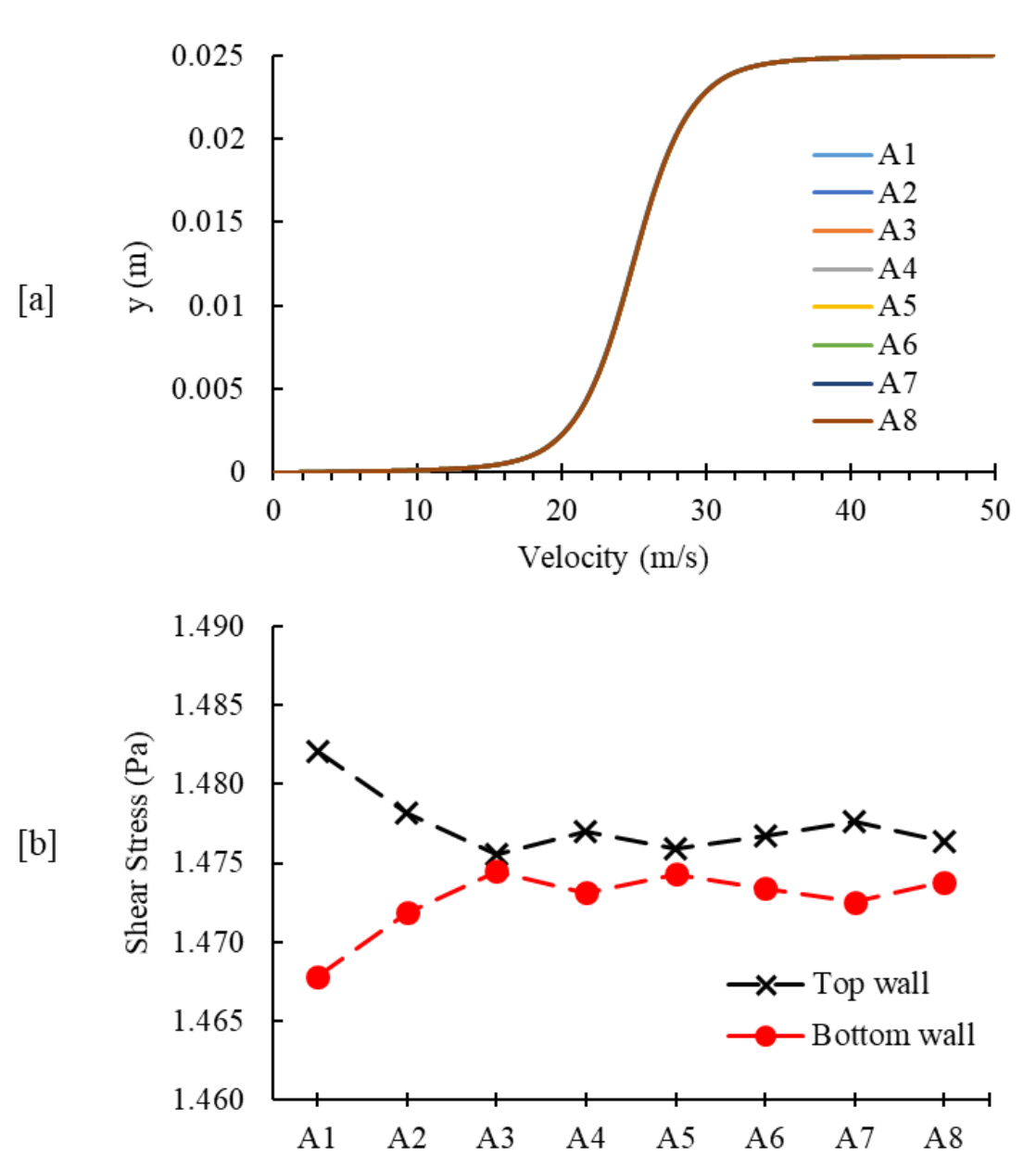

Figure 5. Velocity profile and wall shear stress for simulation set $[\mathrm{A} 1, \mathrm{~A} 2, \ldots, \mathrm{A} 8]$.

\section{Overall Grid Refinement}

The second part of the grid sensitivity study essentially involves the calculation of Grid Convergence Index (GCI). We choose simulation A3 from set A and define it as simulation B0 as the base case for this study. Simulations B-1 and $\mathrm{B} 1$ are performed where $\Delta y_{c}$ is coarsened and refined by a factor of 0.5 respectively. For this study, $y^{+}$is maintained at 0.25. $\Delta x_{c}$ is changed to maintain $A R=1$. The specifics are tabulated in Table 3. Grid Convergence Index for simulation $\mathrm{B}-1, G C I_{B-1}$ was determined to be equal to $0.7 \%$ while the same for B1, GCI $I_{B 1}$ was determined to be $0.05 \%$. GCI values less than $5 \%$ are generally accepted for grid convergence. Hence the results for simulation B0 can be safely considered to be converged.

Table 3. Details of overall grid refinement study.

\begin{tabular}{ccccccc}
\hline Simulation & $y^{+}$ & $r_{\text {inf }}$ & $n$ & $\Delta y_{c}$ & $\Delta x_{c}$ & $G C I$ \\
\hline B-1 & 0.25 & 1.14 & 46 & 0.001 & 0.001 & $0.7 \%$ \\
B0 (= A3) & 0.25 & 1.1 & 55 & 0.0005 & 0.0005 & - \\
B1 & 0.25 & 1.1 & 48 & 0.00025 & 0.00025 & $0.05 \%$ \\
\hline
\end{tabular}


Simulations A1, A2, ., A8 and B-1, B0 and B1 helped acquire a reliable grid to simulate a plane turbulent Couette flow. While the literature suggests that a $y^{+}$of 1 needs to be maintained when Std $k-\varepsilon$ model is used with enhanced wall functions, however, the reduced $y^{+}$is seen to have a further effect, though miniscule, on the higher order variables like shear stress.

\section{TURBULENCE MODEL STUDY}

D1, D2, D3 were simulated to compare the results generated by $S t d k-\varepsilon, S t d k-\omega$ and $k-\omega S S T$ turbulence models respectively. With $S t d k-\varepsilon$ model enhanced wall functions were deployed. Re 21,333 was chosen from Pirozolli et al. [26] as the defining test case for comparison. Mean velocity profiles, noted to be symmetric about the half height, were plotted and compared against data from Pirozolli et al. [26] in Figure 6. The characteristic ' $\mathrm{S}$ ' shaped curve is reproduced by all the turbulence models. Further scrutiny shows that simulations D2 and D3 trace each other's velocity profile while the profile predicted by D1 is slightly more angular and closely follows Pirozzoli et al. [26]. Quantitative difference between the three predictions is apparent on the calculation of the quantity $S=\left.\frac{h}{u_{c}}\left(\frac{d u}{d y}\right)\right|_{y=h}$ (adopted from [1, 26]), which is the slope of the curve at the center of the domain scaled with outer velocity $\left(u_{c}\right)$ units. Pirozolli et al. reported $S=0.0879$ for $R e=21,333$. We see that predictions by D1, D2 and D3 are all slightly less than twice that value reported by Pirozolli et al. [26]. We also investigate inner-velocity $\left(u_{\tau}\right)$ scaled slope $R_{s}=\left.\frac{h}{u_{\tau}}\left(\frac{d u}{d y}\right)\right|_{y=h}$ (adopted from [26]) to compare the results in different light. A similar trend is shown in the values of $R_{s}$ computed by D1, D2 and D3 as was the case with $S$. Though the computed results do not comply with literature, there is an appears a trend, in that D2 and D3 deviate with very small differences and D1 consistently deviates the least with respect to Pirozolli's values.

Table 4. Inner and Outer scaled velocity slope for $\boldsymbol{R} \boldsymbol{e}=21,333$.

\begin{tabular}{ccccr}
\hline & Pirozzoli et al. [26] & D1 & D2 & D3 \\
\hline$S$ & 0.087981 & 0.1605 & 0.1729 & 0.1732 \\
$R_{S}$ & 1.8964 & 3.6378 & 3.8783 & 3.8855 \\
\hline
\end{tabular}

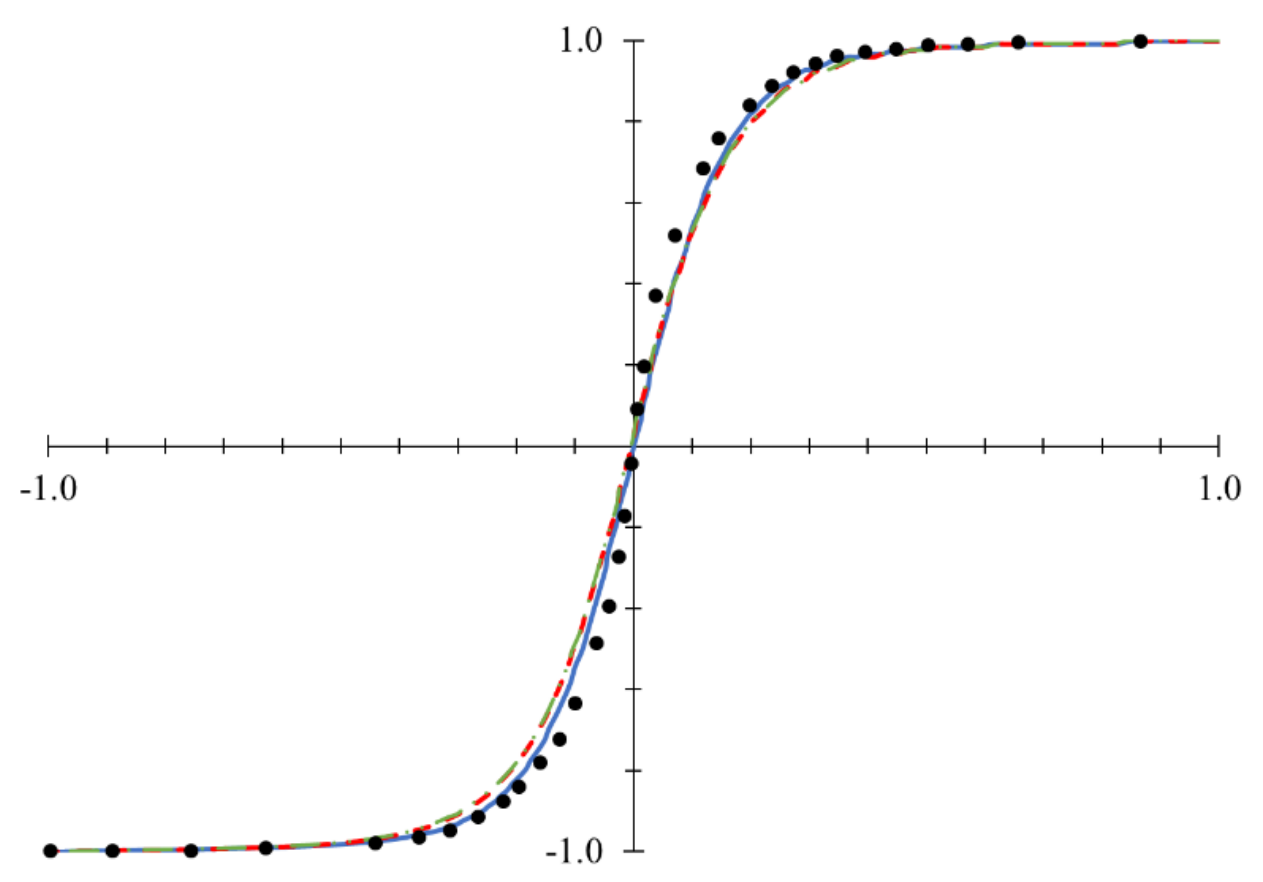

Figure 6. Velocity profile comparison of turbulence models. D1 - Solid blue line. D2 - Dashed red line. D3 - Dash-dot green line. Solid circles - Pirozolli et al. [26].

For a constant pressure incompressible boundary layer flow at Reynolds number close to $10^{6}$, it is known that both the models perform very well, predicting values of friction coefficient and mean velocity within 5\% [28]. For pipe flows 
at $R e=40,000$, the Std. $k-\omega$ model has been shown to give predictions within $6 \%$ of DNS prediction for mean velocities, Reynolds stress and skin friction. However, the model is slightly inaccurate at predicting the value of $k$ near the wall [28]. Table 5 tabulates the near wall performance of turbulence models used in this study. $R e_{\tau}$ and $C_{f}$ predicted by $S t d . k-\omega$ and $k-\omega S S T$ models deviate less compared to the predictions by $S t d . k-\varepsilon$. Both these quantities are derived from the computed wall shear stress indicative of the well-established behavior that $k-\omega$ family models better predict wall bounded flows over free shear flows.

Table 5. Near-wall performance of different turbulence models.

\begin{tabular}{|c|c|c|c|c|c|c|c|c|c|}
\hline \multirow[b]{2}{*}{ Simulation } & \multirow[b]{2}{*}{$\begin{array}{c}\text { Turbulence } \\
\text { model }\end{array}$} & \multirow[b]{2}{*}{$\begin{array}{c}\tau_{w} \\
(\mathrm{~Pa})\end{array}$} & \multirow[b]{2}{*}{$\begin{array}{c}u_{*} \\
(m / s)\end{array}$} & \multicolumn{3}{|c|}{$R e_{\tau}$} & \multicolumn{3}{|c|}{$C_{f}$} \\
\hline & & & & $\begin{array}{l}\text { Ref } \\
{[26]}\end{array}$ & Calculated & Deviation & $\begin{array}{l}\text { Ref } \\
{[26]}\end{array}$ & Calculated & Deviation \\
\hline D1 & Std. $k-\varepsilon$ & 1.48 & 1.099 & 986 & 940 & $4.6 \%$ & 0.00425 & 0.003897 & $8.3 \%$ \\
\hline D2 & Std. $k-\omega$ & 1.51 & 1.110 & 986 & 950 & $3.6 \%$ & 0.00425 & 0.003976 & $6.4 \%$ \\
\hline D3 & $k-\omega S S T$ & 1.51 & 1.110 & 986 & 950 & $3.6 \%$ & 0.00425 & 0.003976 & $6.4 \%$ \\
\hline
\end{tabular}

Figure 7 shows the kinetic energy with respect to $y^{+}$. The normal Reynolds stress components $\left(\overline{u^{\prime 2}}, \overline{v^{\prime 2}}, \overline{w^{\prime 2}}\right)$ normalized by the square of the friction velocity presented in Pirozzoli et al. [26] were added and then divided by 2 to give the quantity $k / u_{*}^{2}$. The simulated kinetic energy, $k$ was directly extracted from simulations D1, D2 and D3 and normalized by $u_{*}^{2}$. The normalized kinetic energy is seen to peak at $y^{+} \approx 20$ in [26] consistent with behavior of kinetic energy observed in channel flows. The normalized kinetic energy predicted by D1 is off by $46 \%$ at the peak while that predicted by D2 and D3 are off by $61 \%$. This difference in prediction of kinetic energy may stem from two factors: 1 ) The difference in turbulence model used, 2) Substituting a 2D computation model for a 3D flow. While Std. $k-\varepsilon$ with enhanced wall functions model predicts a wide range of flows satisfactorily, since it uses a Boussinesq eddy-viscosity concept which assumes isotropic turbulent viscosity, which is known to fail in reproducing flows which arise due to unequal normal Reynolds stresses [32]. Std. $k-\omega$ is criticized that it does not predict the distribution of $k$ and $\varepsilon$ accurately. Data represented in Figure 7 is consistent with this criticism [29]. While it is also known that Std. $k-\varepsilon$ over predicts the production of turbulence in highly strained flows [32], Figure 7 suggest that the peak and the plateau qualitatively follow data presented by Pirozzoli et al. [26]

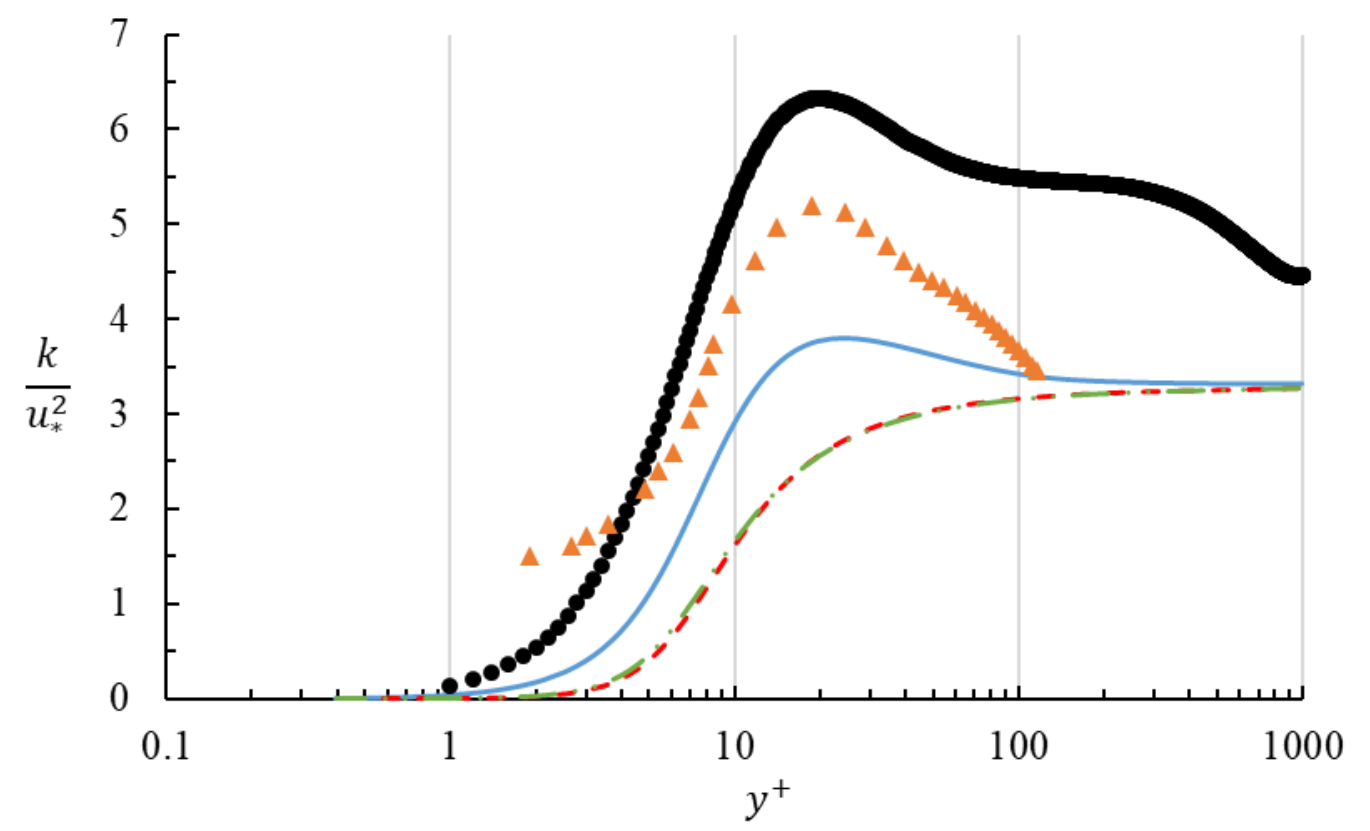

Figure 7. Normalized turbulent kinetic energy. Solid orange triangle - El Telbany and Reynolds [5]. Refer to Figure 6 for current study's legend.

While there is a lot of literature suggesting that $S t d k-\omega$ is best suited for high shear internal flows, we can determine from comparing the results of simulations D1, D2 and D3 that Std. $k-\varepsilon$ with enhanced wall functions is best suited for the current simulations of turbulent Couette flow. 


\section{VALIDATION AND DISCUSSION}

Simulations E1, E2 and E3 are utilized to determine if $S t d k-\varepsilon$ with enhanced wall functions is successful in simulating a range of Reynolds numbers in turbulent Couette flow. Results from simulation F1 are then utilized to compare with those of E1, E2 and E3. Figure 8 shows the velocity profile across the height of the channel for Reynolds numbers 3000, 10133 and 21333. From Pirozolli's data we see that the normalized velocity profiles come very close to each other in the middle of the channel, while they become broad and fuller near the walls with increasing Reynolds number. This observation is consistent with characteristics of turbulent boundary layer. The profiles generated by the simulations closely follow Pirozolli's data. Upon a critical examination, the agreement is observed to decrease slightly in the middle of the channel for higher Reynolds numbers. However, it is reassuring that the core flow becomes fuller and occupies approximately $60 \%$ of the domain at $R e=3,000$ while almost $75-80 \%$ at $R e=21,333$. Further, the trend is maintained for flow at $R e=51,099$, where the core region becomes wider and occupies almost $90 \%$ of the flow domain.

Mean velocity slopes at the center of the channel as a function of $R e$ are shown in Figure 9. Busse [33] suggests that the centerline velocity slope should asymptote to a constant with increasing $R e$, typically $S \approx 0.2$. While the values of $S$ from the current study, though very close to 0.2 , there seems to be no trend to asymptote to this limit. However, simulations E2, E3 and F1 which could be considered moderately high $R e$ simulations do tend to follow the trend described by Robertson's [1] empirical formula $S=\frac{0.78}{\ln (R e)}$. It may be noted that DNS data by Pirozolli et al., though decreasing in trend, predicted values much less than the available literature while on the other hand, the experimental data from El Telbany and Reynolds [5], predicted values more than Robertson's empirical formula, although their data seems to tend towards $S=0.2$. Robertson also recorded that the parameter $S$ was very sensitive to minor changes in the flow, which is evident from examining Figure 8 since the velocity profile from DNS and E1, E2 and E3 agree very well with each other but values of $S$ do not. According to some studies (Townsend [34]) the quantity, $R_{s}=\frac{h}{u_{\tau}}\left(\frac{d u}{d y}\right)$ should approach a limit as $R e$ increases. In this regard, $R_{s}$ values of simulations E2, E3 and F1 tend to remain constant at 3.6, with $R_{S}$ value of smallest Reynolds number simulation, E1 being lower by $18 \%$ from the constant. Experimental studies of El Telbany and Reynolds [5] and Kitoh et al. [35] as well as DNS studies of Pirozolli et al. [26] do not show any asymptote behavior of $R_{s}$ and differ largely by their absolute value as well as the trend they exhibit with respect to increasing $R e$. There does not seem to be enough agreement on the values of $R_{S}$ considering different studies.

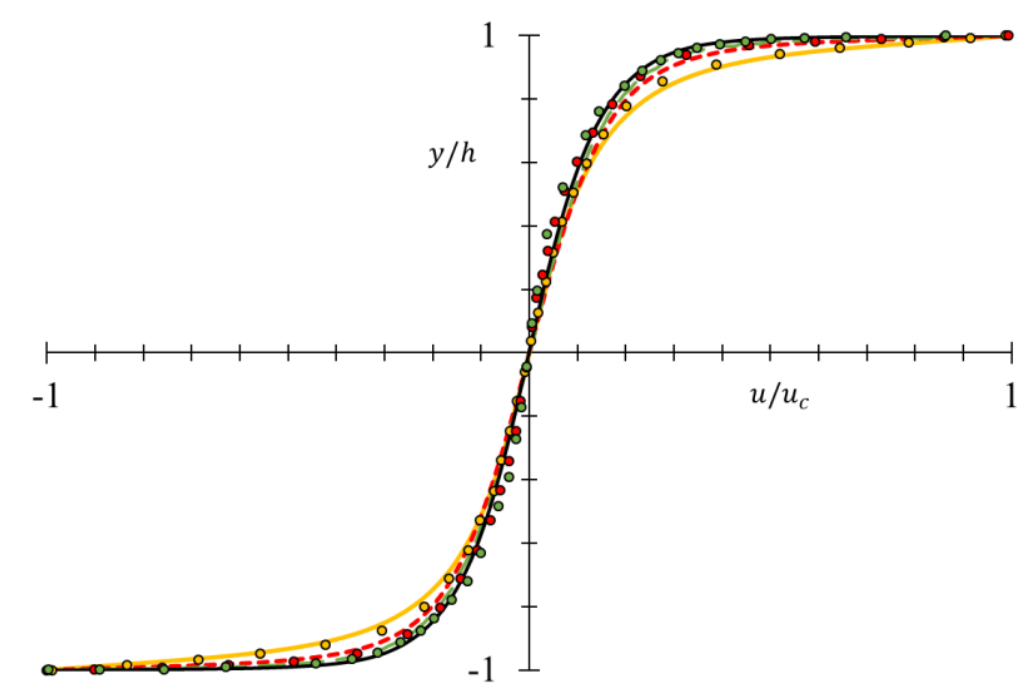

Figure 8. Normalized velocity profiles for different Reynolds number. Current study represented by coloured lines: E1 - yellow; E2 - red; E3 - green; F1 - solid black line. Solid circles represent data from Pirozolli et al.[26]: Re 3,000 yellow; Re 10,133 - red; Re 21,333 - green. 

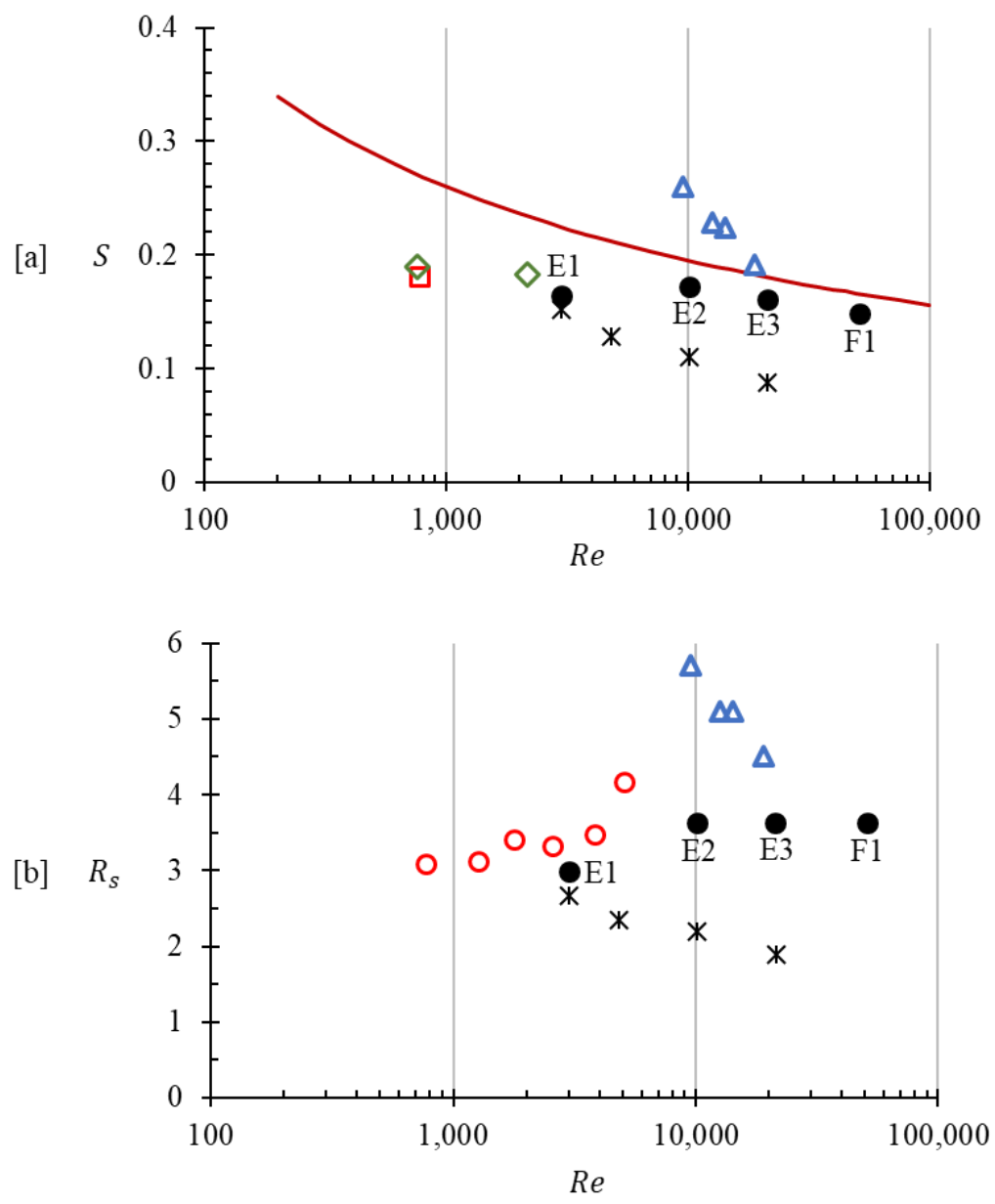

Figure 9. Slopes of mean velocity at the centre of the flow domain scaled by - a) outer velocity: $\boldsymbol{h} / \boldsymbol{u}_{\boldsymbol{c}} ;$ b) inner velocity: $\boldsymbol{h} / \boldsymbol{u}_{*}$ vs $\boldsymbol{R e}$. Data from current study [E1, E2, E3 and F1] is represented by solid black circles. Black cross marks denote data from Pirozolli et al. [26]. Open symbols denote experimental data (extracted from Pirozolli et al. [26]): Komminaho et al. [36] - red square; Tsukahara et al. [37] - green diamonds; El Telbany and Reynolds [5] - blue triangles; Kitoh et al. [35] - red circles. The solid red line represents the fit $\boldsymbol{S}=\mathbf{0 . 7 8} / \mathbf{l n}(\boldsymbol{R} \boldsymbol{e})$ - Robertson [1].

While the core velocity profiles and slopes are encouraging, the inner velocity profiles also show excellent agreement with the law of the wall. Figure 10 shows the log-law comparison for E1, E2 and E3. The velocity profiles resolved for all the Reynolds numbers show good agreement with the log law defined by the constants, $k=0.39$ and $C=5.1$. We find that the computational parameters chosen predict velocity profiles with a range of Reynolds numbers from 3,000 to 21,333 and when extended to $R e=51,099$ the simulation F1 is successful in generating good agreement with the log-law indicating that the enhanced wall functions consistently predict dependable results, at least for the range of Reynolds number tested in this study.

Some comments on the variation of core kinetic energy with Reynolds numbers was discussed by Andersson and Pettersson [8]. They used an Algebraic Reynolds stress model (ASM), which was a simplified form of the Reynolds stress transport model (RSM), a second moment closure proposed by Launder and Shima [38] with a new near-wall damping function. They found that the model does not fulfill the underlying assumptions of ASM for lower Reynolds numbers, but was in excellent agreement for higher Reynolds numbers. Figure 11 may be indicative of that behavior as we see that the values for $R e<2,000$, the normalized kinetic energy shoots up by around $12 \%$ but remains practically constant for $R e \geq 2,000$. While the ASM is a slightly higher fidelity simulation compared to the family of two-equation models, we find that the data from the current study agrees with a deviation of just about $6 \%$. The trend of constancy of the normalized kinetic energy in the core region is replicated and gives confidence in our predictions of the highest Reynolds number simulated in the study. 


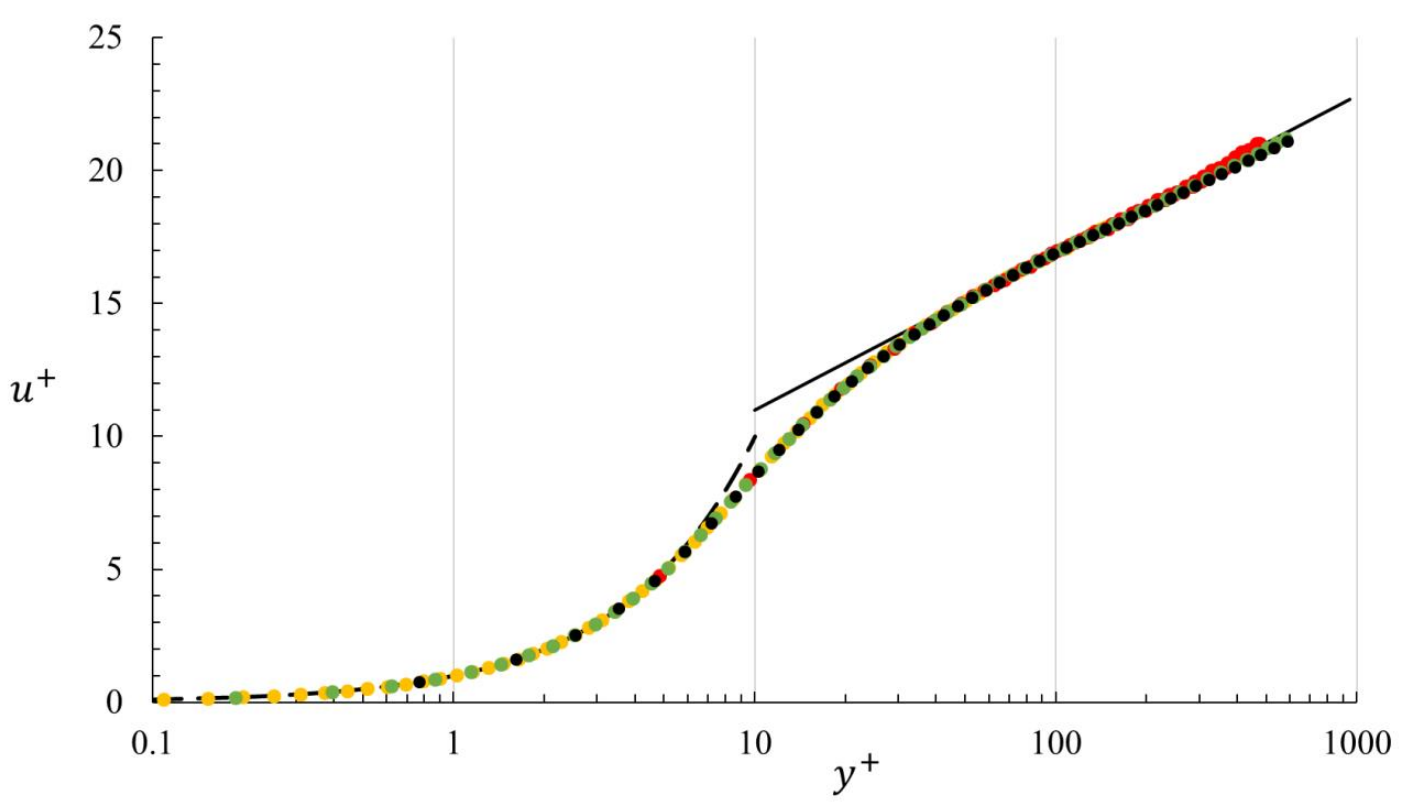

Figure 10. Log law comparisons for simulation set [E1-E3]. Coloured circles represent current study's data: $\boldsymbol{R} \boldsymbol{e} 3,000-$ yellow; $\boldsymbol{R} \boldsymbol{e} 10,133$ - red; $\boldsymbol{R} \boldsymbol{e}$ 21,333 - green; $\boldsymbol{R} \boldsymbol{e}$ 51,099-black. Dashed black line represents $\boldsymbol{y}^{+}=\boldsymbol{u}^{+}$. Solid black line represents log-law with constants $\boldsymbol{k}=0.39$ and $\boldsymbol{C}=5.1$

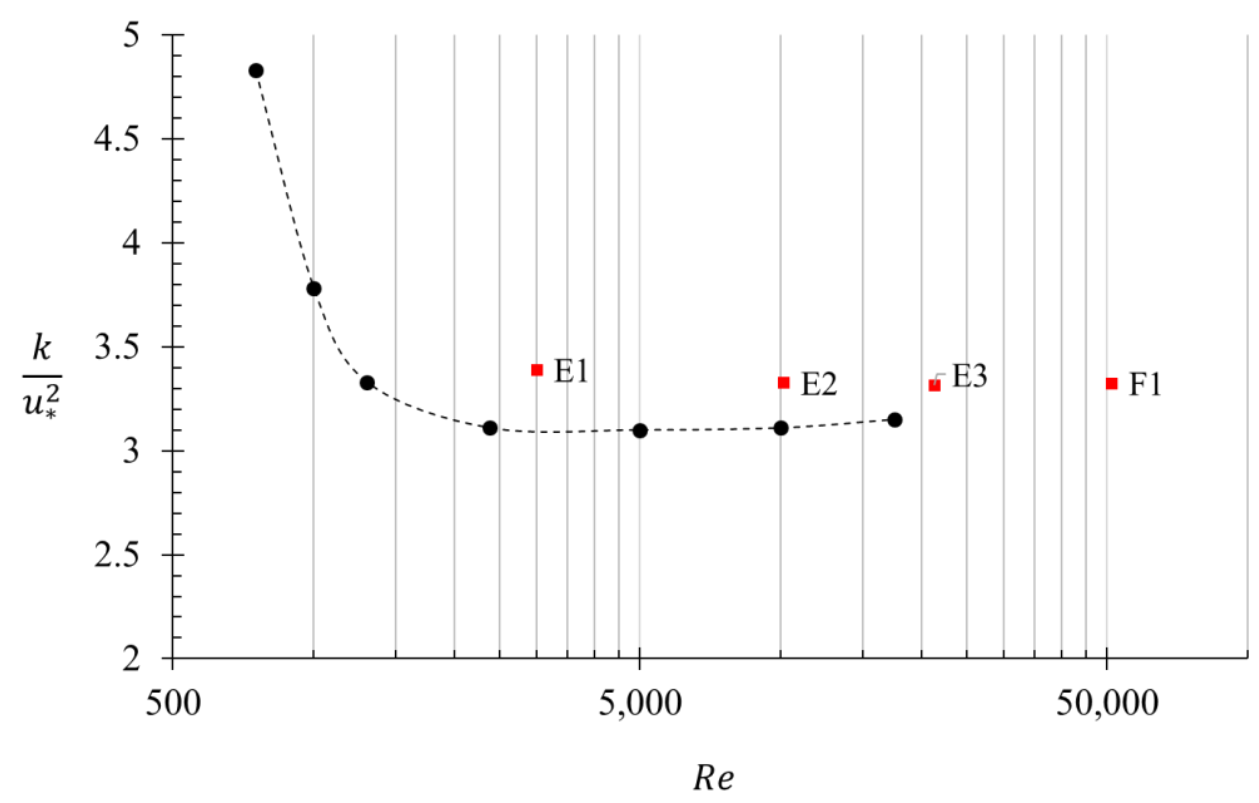

Figure 11. Normalized centreline kinetic energy against Reynolds number. Black dots and dashed line represent computational data from the Reynolds stress transport model published by Andersson and Pettersson [8]. Red squares represent current study.

\section{Skin friction Coefficient}

While the simulated velocity and shear stress profiles separately show good agreement with [26], comparison of skin friction coefficient helps us to look at their effects together with the Reynolds number. The skin friction coefficient gives a direct relation between the Reynolds number, shear stress and flow velocity, thus making it possible to analyze Couette flow in a wholesome manner. There is a vast amount of experimental data expounding the relation between the skin friction coefficient and Reynolds number. All the solid markers in Figure 12 show some of the experimental data available on the topic, and it is evident that there is a dearth of study for $R e>20,000$. Reichardt [2] first published experimental data for the skin friction coefficient which was referenced by Robertson [1] who suggested a friction law, 


$$
C_{f}=\frac{G}{\log R e_{c}^{2}}
$$

with the proposed constant $G=0.383$. We see that the results from current study align perfectly well with $G=0.383$. Many values for this constant have been proposed varying slightly in the range of 0.351 (El Telbany and Reynolds [5]) to 0.420 (Tsukahara et al [37]) and so we see their data fall on both the sides of the black curve. There also have been many DNS studies by the likes of Bech [39], Tsukahara [37] including Pirozolli et al. [26] -their results plotted in Figure 12. It is interesting to note that while experimental results have varied on both sides of the curve, all DNS data consistently showed a higher value of $C_{f}$, corresponding to $G \approx 0.420$. Using the $\log$-law representation of the mean velocity profile, Schlichting and Gertsen [40] proposed the following relation,

$$
\left(\frac{2}{C_{f}}\right)^{1 / 2}=\frac{1}{k} \log \left(\operatorname{Re}_{c}\left(\frac{C_{f}}{2}\right)^{1 / 2}\right)+C+\bar{C}
$$

where $C$ and $\bar{C}$ are constants. Pirozolli et al. [26] proposed to fit their data with $k \approx 0.41 C \approx 5$ and $\bar{C}=0$. We take the same values from log-law fit $-k=0.39, C=5.1$ and $\bar{C}=0$. We see that this fit agrees with results of all the Reynolds numbers simulated including the highest one of this study.

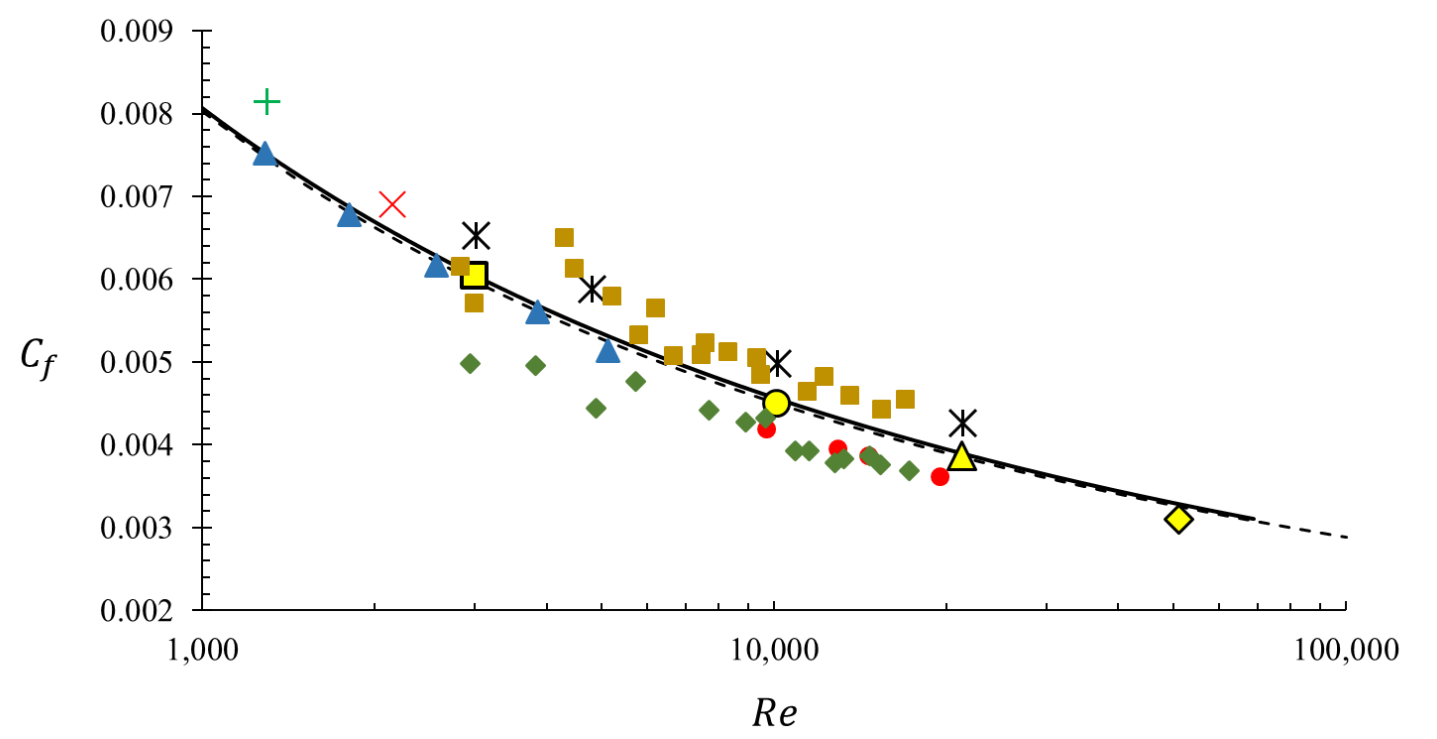

Figure 12. Skin friction coefficient vs Reynolds number - Yellow symbols with black border denote results from current study: E1 - square. E2 - circle. E3 - triangle. F1 - diamond. Solid figures denote experimental data (extracted from Pirozolli et al. [26]): Kitoh et al. [35] (triangles). El Telbany \& Reynolds [5] (circles). Reichardt [2] (diamonds). Robertson [1] (squares). Intersecting symbols denote DNS data: Bech et al. [39] (plus). Tsukahara et al. [37] (cross). Pirozolli et al. [26] (stars). The solid line indicates correlation by Schlicting \& Gertsen [40] with $\boldsymbol{k}=0.39, \boldsymbol{C}=5.1$ and $\overline{\boldsymbol{C}}=0$. Dashed line indicates the logarithmic friction law with $\boldsymbol{G}=0.383$.

Figures $8-12$ show with evidence that $S t d k-\varepsilon$ applied with enhanced wall functions consistently produces reliable computational results for Reynolds numbers ranging from 3,000 to 21,333 coherent with available literature on the topic. When extended to the highest Reynolds number simulated in the study, $R e=51,099$, the results agreed very well with the trends set by lower Reynolds number that were validated against literature.

\section{CONCLUSION}

This study can be thought to be divided into two parts. In the first part Std $k-\varepsilon$ applied with enhanced wall functions was determined to be most reliable to simulate high Reynolds number turbulent Couette flow. In the process, contrary to literature on turbulence modelling on wall-bounded flows, it was seen that embedding more grid points in the viscous region of the turbulent boundary layer under the prescribed $y^{+}=1$ may achieve more reliable higher order variables. Literature on turbulence modeling indicates that $S t d k-\varepsilon$ is better at predicting free shear flows at high Reynolds numbers while $S t d k-\omega$ and its variants are better at predicting wall bounded flows. In the current study however, it 
was observed that the core region of Couette flow was better predicted by $\operatorname{Std} k-\varepsilon$ in terms of closer agreement of centerline slopes and velocity profiles, while the near-wall performance of Std $k-\omega$ and SST models was only slightly better in terms of wall shear stress and friction velocity. Std $k-\varepsilon$, however, predicted the prominent and well-known near-wall peak of turbulent kinetic energy consistent with DNS and experimental data, though the absolute value of the kinetic energy at the peak was much smaller. In the second part, Std $k-\varepsilon$ with enhanced wall functions was deemed to be consistently efficient, robust and reliable over the range of Reynolds numbers from 3,000 to 21,333. The simulations showed good agreement when velocity profiles, velocity slopes and kinetic energy at the center-line, log-law fit, and the Reynolds number dependence of skin friction coefficient were compared with available literature. This result was then used to simulate a flow with $\operatorname{Re} 51,099$, which continued to predict the trend exhibited by the lower Reynolds numbers consistently, in all gross variable comparisons.

\section{NOTATION}

$y^{+} \quad$ Dimensionless distance to the wall

$\Delta y_{1} \quad$ Height of the first layer, counted from the wall, $\frac{y^{+} \mu}{u_{*} \rho}$

$\Delta x_{c} \quad$ Length of the characteristic grid cell

$\Delta y_{c} \quad$ Height of the characteristic grid cell

$n \quad$ Number of inflation layers, $n=\frac{\ln \left(\frac{\Delta y_{c}}{\Delta y_{1}}\right)}{\ln \left(r_{i n f}\right)}$

Inflation growth ratio, defined as ratio of $r_{\text {inf }}$

$A R$

$h$

$R e_{\tau}$

$L_{x}$

$R_{S}$ heights of successive inflation layers

Aspect ratio of the grid cell, $\frac{\Delta x_{c}}{\Delta y_{c}}$

Channel half height

Reynolds number based on $u_{*}, \frac{u_{*} h}{v}$

Length of the computational domain

Inner-velocity scaled slope, $\frac{h}{u_{\tau}}\left(\frac{d u}{d y}\right)$ $\begin{array}{cl}u_{c} & \text { Centerline velocity } \\ u_{w} & \text { Velocity of plate, } 2 u_{c} \\ \mu & \text { Dynamic viscosity } \\ v & \text { Kinematic viscosity, } \frac{\mu}{\rho}\end{array}$

$C_{f} \quad$ Skin friction coefficient, $\frac{\tau_{w}}{\frac{1}{2} \rho u_{c}^{2}}$

$\tau_{w} \quad$ Wall shear stress

$\rho \quad$ Density of the medium

Re Reynolds number, $\frac{u_{c} h}{v}$

$u_{*} \quad$ Friction velocity, $\sqrt{\frac{\tau_{w}}{\rho}}$

$S \quad$ Outer-velocity scaled slope, $\frac{h}{u_{c}}\left(\frac{d u}{d y}\right)$

\section{REFERENCES}

[1] J. M. Robertson, "On Turbulent Plane-Couette Flow," in Proceedings of the sixth Midwestern Conference on Fluid Mechanics, 1959, pp. 169-182.

[2] H. Reichardt, "Über die Geschwindigkeitsverteilung in einer geradlinigen turbulenten Couetteströmung," Zeitschrift für Angew. Math. und Mech., vol. 36, pp. 26-29, 1956.

[3] H. J. Leutheusser and V. H. Chu, “Experiments on Plane Couette Flow,” J. Hydraul. Div., vol. 97, no. 9, pp. $1269-1284,1971$.

[4] M. Aydin and H. J. Leutheusser, "Novel experimental facility for the study of plane Couette flow," Rev. Sci. Instrum., vol. 50, no. 11, pp. 1362-1366, 1979, doi: 10.1063/1.1135726.

[5] M. M. M. El Telbany and A. J. Reynolds, “The Structure of Turbulent Plane Couette Flow," J. Fluids Eng., vol. 104, no. 3, p. 367, 2009, doi: 10.1115/1.3241853.

[6] N. Tillmark and P. H. Alfredsson, "Experiments on transition in plane Couette flow," J. Fluid Mech., vol. 235, no. HY9, pp. 89-102, 1992.

[7] F. S. Henry and A. J. Reynolds, "Analytical Solution of Two Gradient-Diffusion Models Applied to Turbulent Couette Flow," J. Fluids Eng., vol. 106, no. 2, p. 211, 2009, doi: 10.1115/1.3243104.

[8] H. I. Andersson and B. A. Pettersson, "Modeling plane turbulent Couette flow," Int. J. Heat Fluid Flow, vol. 15, no. 6, pp. 447-455, 1994, doi: 10.1016/0142-727X(94)90003-5.

[9] S. Nisizima and A. Yoshizawa, "Turbulent channel and Couette flows using an anisotropic k-epsilon model," Am. Inst. Aeronaut. Astronaut. J., vol. 25, no. 3, pp. 414-420, 2008, doi: 10.2514/3.9639. 
[10] J. C. Monnier and M. Stanislas, "Critical examination of various turbulence models applied to different one-dimensional internal flows," in Proceedings of the Sixth International Conference on Numerical Methods in Laminar and Turbulent Flow, 1989, pp. 297-306.

[11] H. S. Zhang, R. M. C. So, and M. L. Zhu, "A near-wall second-order turbulence model for wall-bounded flows," in Ninth Symposium of Turbulent Shear Flows, 1993, pp. 8.2.1-8.2.6.

[12] H. Sund, "Validation of a second-moment closure to low Reynolds number plane Couette flow," in Proceedings of the Eighth International Conference on Numerical Methods in Laminar and Turbulent Flow, 1993, pp. 241-248.

[13] L. S. Tuckerman, M. Chantry, and D. Barkley, "Patterns in Wall-Bounded Shear Flows," Annu. Rev. Fluid Mech., vol. 52, no. 1, pp. 343-367, Jan. 2020, doi: 10.1146/annurev-fluid-010719-060221.

[14] M. J. Lee and J. Kim, "The structure of turbulence in a simulated plane Couette flow," in 8th Symposium on Turbulent Shear Flows; Sept. 9-11, 1991, pp. 5.3.1-5.3.5.

[15] A. Pershin, C. Beaume, and S. M. Tobias, "Dynamics of spatially localized states in transitional plane Couette flow," J. Fluid Mech., 2019, doi: 10.1017/jfm.2019.154.

[16] B. Eckhardt and S. Zammert, "Small scale exact coherent structures at large Reynolds numbers in plane Couette flow," Nonlinearity, Oct. 2017, doi: 10.1088/1361-6544/aa9462.

[17] D. Olvera and R. R. Kerswell, "Exact coherent structures in stably stratified plane Couette flow," J. Fluid Mech., 2017, doi: 10.1017/jfm.2017.447.

[18] T. Kawata and T. Tsukahara, "Numerical investigation on scale-by-scale Reynolds stress transport in plane Couette turbulence," in 11th International Symposium on Turbulence and Shear Flow Phenomena, TSFP 2019, 2019.

[19] T. Kawata and P. H. Alfredsson, "Inverse Interscale Transport of the Reynolds Shear Stress in Plane Couette Turbulence," Phys. Rev. Lett., 2018, doi: 10.1103/PhysRevLett.120.244501.

[20] P. R. Spalart, A. Garbaruk, and M. Strelets, "RANS Solutions in Couette flow with streamwise vortices," Int. J. Heat Fluid Flow, 2014, doi: 10.1016/j.ijheatfluidflow.2014.04.010.

[21] J. Guo, "General Mean Velocity Distribution Law for Smooth-Wall Plane Couette Flow," J. Eng. Mech., vol. 144, no. 1, p. 04017146, Jan. 2018, doi: 10.1061/(ASCE)EM.1943-7889.0001370.

[22] K. Duraisamy, G. Iaccarino, and H. Xiao, “Turbulence Modeling in the Age of Data," Annu. Rev. Fluid Mech., Mar. 2018, doi: 10.1146/annurev-fluid-010518-040547.

[23] M. Kaandorp, "Machine Learning for Data-Driven RANS Turbulence Modelling," Delft Univ. Technol., 2018.

[24] S. Hoyas and J. Jiménez, "Scaling of the velocity fluctuations in turbulent channels up to Ret=2003," Phys. Fluids, 2006, doi: $10.1063 / 1.2162185$.

[25] E. V Mortikov, A. V Glazunov, and V. N. Lykosov, "Numerical study of plane Couette flow: turbulence statistics and the structure of pressure-strain correlations," Russ. J. Numer. Anal. Math. Model., vol. 34, no. 2, pp. 119-132, Apr. 2019, doi: 10.1515/rnam-2019-0010.

[26] S. Pirozzoli, M. Bernardini, and P. Orlandi, "Turbulence statistics in Couette flow at high Reynolds number," J. Fluid Mech., vol. 758, pp. 327-343, 2014, doi: 10.1017/jfm.2014.529.

[27] S. A. Orszag, "Analytical theories of turbulence," J. Fluid Mech., vol. 41, no. 2, pp. 363-386, 1970, doi: $10.1017 /$ S0022112070000642.

[28] I. B. (Wester. V. U. Celik, "Introductory turbulence modeling," 1999.

[29] F. R. Menter, "Two-equation eddy-viscosity turbulence models for engineering applications," Am. Inst. Aeronaut. Astronaut. J., vol. 32, no. 8, pp. 1598-1605, 1994, doi: 10.2514/3.12149.

[30] S. B. Pope, Turbulent Flows. New York: Cambridge University Press, 2000.

[31] "Near-Wall Treatments for Wall Bounded Turbulent Flows," 2009. .

[32] G. Biswas and V. Eswaran, Turbulent Flows: Fundamentals, Experiments and Modeling, 1st ed. Narosa, 2002.

[33] F. H. Busse, "Bounds for turbulent shear flow," J. Fluid Mech., vol. 41, no. 1, pp. 219-240, 1970, doi: $10.1017 /$ S0022112070000599.

[34] A. A. Townsend, The Structure of Turbulent Shear Flow, 2nd ed. Cambridge University Press, 1976.

[35] O. Kitoh, K. Nakabayashi, and F. Nishimura, "Experimental study on mean velocity and turbulence characteristics on plane Couette flow: low-Reynolds-number effects and large longitudinal vortical structures," J. Fluid Mech., pp. 199-227, 2005.

[36] J. Komminaho, A. Lundbladh, and A. V Johansson, "Very large structures in plane turbulent Couette flow," J. Fluid Mech., vol. 320, pp. 259-258, 1996.

[37] T. Tsukahara, H. Kawamura, and K. Shingai, "DNS of turbulent Couette flow with emphasis on the large-scale structure in the core region," J. Turbul., vol. 7, pp. 1-16, 2006.

[38] B. E. Launder and N. Shima, "Second-moment closure for the near-wall sublayer: development and application," Am. Inst. Aeronaut. Astronaut. J., vol. 27, pp. 1319-1325, 1989.

[39] K. H. Bech, N. Tillmark, P. H. Alfredsson, and H. I. Andersson, "An investigation of turbulent plane Couette flow at low Reynolds numbers,” J. Fluid Mech., vol. 286, pp. 291-325, 1995.

[40] H. Schlichting and K. Gersten, Boundary Layer Theory, 8th ed. Springer, 2000. 